\title{
Risk Factors Associated with an Increased Risk of Deep Sternal Wound Infections in Patients After Coronary Artery Bypass Grafting and Heart Defect Surgery
}

\author{
Migle Vitartaite, ${ }^{1}$ Donata Vaičiulytè, ${ }^{1}$ Jonė Venclovienè, ${ }^{2}$ Edmundas Širvinskas, ${ }^{3}$ Rasa Bukauskienė ${ }^{3}$ \\ Povilas Jakuška ${ }^{3}$, Renata Vimantaite ${ }^{4}$ \\ ${ }^{1}$ Faculty of Medicine, Medical Academy, Lithuanian University of Health Sciences, Kaunas, Lithuania; \\ ${ }^{2}$ Institute of Cardiology, Lithuanian University of Health Sciences, Kaunas, Lithuania; \\ ${ }^{3}$ Lithuanian University of Health Sciences Kaunas Clinics Department of Cardiac, Thoracic and Vascular Surgery, Kaunas, Lithuania; \\ ${ }^{4}$ Lithuanian University of Health Sciences Kaunas Clinics Faculty of Nursing, Kaunas, Lithuania
}

\section{ABSTRACT}

Background: Despite improvements over time with regard to morbidity, mortality, and long-term survival, deep sternal wound infection (DSWI) continues to be a major complication after open-heart surgery. This is why it is important to identify possible risk factors for postoperative development of DSWI in patients undergoing coronary artery bypass grafting and valve replacement. The aim of this study was to identify the risk factors for postoperative development of deep sternal wound infection in patients after coronary artery bypass grafting and heart defect surgery at the Department of Thoracic, Cardiac, and Vascular Surgery of the Hospital of Lithuanian University of Health Sciences.

Methods: This retrospective study analyzed 201 patients, who underwent coronary artery bypass grafting and heart defect surgery between January 2017 and December 2018. The case group contained 45 patients, who had to be reoperated because of deep sternal wound infection, and the control group consisted of 156 randomly selected patients. For descriptive statistics, we used means, median values, ranges, standard deviations, and $95 \%$ confidence intervals, where appropriate. Categorical data were analyzed using the chisquare or Fisher's exact test. Student T-test and Mann-Whitney used to compare numerical variables. Logistic regression model adjusting for age and gender was used to compare the risk of infection. A P-value of $<0.05$ was considered to be statistically significant. SPSS 26.0 was used for calculations.

Results: Logistic regression analysis revealed that independent risk factors for sternal wound infection were high BMI (odds ratio [OR] 1.15, CI 1.06-1.24), preoperative CRP (OR 1.08, CI 1.01-1.16), long duration of cardiopulmonary

Received April 29, 2021; received in revised form fune 3, 2021; accepted fune 3, 2021.

Correspondence: Migle Vitartaite, Faculty of Medicine, Medical Academy, Lithuanian University of Health Sciences, Kaunas, Lithuania (e-mail: migle. vitartaite@stud.lsmu.lt). bypass (OR 1.02, CI 1.01-1.03), intraoperative anemia (OR 0.97, CI 0.95-0.99), and postoperative CRP concentration (OR 1.10; CI 1.05-1.16).

Conclusions: Preoperative assessment to identify obese individuals as being at risk and techniques to minimize the duration of surgery and intraoperative blood loss may help reduce postoperative deep sternal wound infections.

\section{INTRODUCTION}

Coronary artery bypass grafting (CABG) is an effective treatment option for coronary artery disease [Varma 2019]. Valvular heart diseases successfully are treated surgically as well [Taylor 2012]. Although cardiac surgeries are considered to be clean since no contaminated viscera are opened during surgery [Vos 2018], the patients still are at risk of developing wound infections [Taylor 2012; Vos 2018]. Wound infection following cardiac surgery can be superficial or deep sternal infection, which also is known as mediastinitis [Yumun 2014]. Deep sternal wound infection is a life-threatening complication that is associated with high morbidity, mortality, prolonged length of hospital stay, and increased cost of healthcare [Lemaignen 2015; Sá 2017]. The incidence of deep sternal wound infection varies from $0.4 \%$ to $5 \%$ [Badawy 2014]. Early diagnosis of mediastinitis and aggressive treatment could reduce mortality after cardiac surgery [Kubota 2013]. Many authors have investigated the risk factors that are associated with the development of deep sternal wound infection after cardiac surgery. According to the literature, risk factors include female sex, older age, diabetes, renal failure, obesity, chronic obstructive pulmonary disease, duration of surgery, re-exploration for bleeding, blood transfusion, prolonged ventilation, and postoperative vasopressive support [Lemaignen 2015; Vos 2018]. The aim of this study is to identify the risk factors for postoperative development of deep sternal wound infection in patients after coronary artery bypass grafting and heart defect surgery at the Department of Thoracic, Cardiac and Vascular Surgery of Lithuanian University of Health Sciences. 


\section{MATERIAL AND METHODS}

During January 2017 and December 2018 at the Department of Thoracic, Cardiac and Vascular Surgery of the Hospital of Lithuanian University of Health Sciences, 1936 patients underwent coronary artery bypass grafting and heart defect surgery. This retrospective study analyzed 201 patients, who underwent CABG and heart defect surgery, during a two-year period. The case group contained 45 (out of 1936) patients who had to be reoperated because of deep sternal wound infection, and the control group consisted of 156 (out of 1891) randomly selected patients. We collected preoperative data, including sex, age, BMI, type of surgery, complete blood count (CBC) and biochemical blood test results, and intraoperative data that included duration of surgery, duration of cardiopulmonary bypass, the need of vasopressors, inotropes, diuretic, infusion therapy, blood transfusion, hemorrhage, $\mathrm{CBC}$, and biochemical blood test and blood gas results. We also collected postoperative data that included duration of mechanical ventilation, antibiotic use, the need for dialysis, diuretics, vasopressors, inotropes, infusion therapy, blood transfusion, $\mathrm{CBC}$ and biochemical blood test and blood gas results, and lengths of hospital and ICU stay. We did not include blood glycemic levels because normoglycemia is maintained during surgery, after surgery in the ICU with rapid-acting insulin, and in the hospital ward with everyday patients' medicines for hyperglycemia.

Statistical analysis was performed using the SPSS 26.0 (Statistical Package for Social Science for Windows 19) statistical software package. Quantitative variables were described as mean and standard deviation (SD). The Shapiro-Wilks and Kolmogorov-Smirnov tests were used for the determination of quantitative data distribution. When the distribution of variables was normal, Student's t-test was used for comparison of quantitative sizes of two independent samples, and the non-parametric Mann-Whitney $\mathrm{U}$ test was used to compare non-normally distributed numerical variables. Categorical data were compared between the two groups, using the chi-square (c2) test or Fisher's exact test. Linear dependence between variables was evaluated using correlation coefficients. Pearson's or Spearman's correlation coefficient was used taking into account the distribution of variables. $P$-value of $<0.05$ was considered to be statistically significant. Logistic regression model adjusting for age and gender was used to compare the risk of infection.

\section{RESULTS}

Neither mean age $(69.5 \pm 9.3$ vs. $67.6 \pm 10.1 ; P=0.259)$ nor sex ( $79 \mathrm{~W}$ vs. $122 \mathrm{M} ; P=0.559)$ was statistically different between the case and control groups.

Preoperative patient characteristics and pre-, peri- and postoperative variables were evaluated as predictors of DSWI. (Table 1) This analysis showed that in preoperative evaluation mean BMI $(P=0.001)$, mean CRP $(P=0.018)$, mean creatinine $(P=0.04)$, and a number of patients having diabetes mellitus $(P=0.01)$ can be acknowledged as risk factors for DSWI. No difference was seen between the groups in age, sex, the concentration of leukocytes, neutrophils, mean $\mathrm{Hb}$, and cardiac operation in anamnesis. As for intraoperative variables, mean time of duration of surgery $(P=$ $0.002)$, cardiopulmonary bypass $(P=0.002)$, and aortic crossclamp time $(P=0.023)$ had a significant difference, when comparing the two groups. Higher anemia during surgery in the case group $(P<0.001)$ did not lead to more frequent blood transfusion. Contrary to that, blood transfusion was more frequently performed on patients in the control group $(P=0.014)$. Post-operation, just as we expected, mean leukocytes $(P=0.001), \mathrm{CRP}(P<0.001)$, days of taking antibiotics $(P<0.001)$, and mean length of stay in the ICU $(P=0.01)$ was more frequent or higher in the group where patients experienced DSWI. On the other hand, delirium was more common amongst the patients in the control group $(P=$ $0.001)$. Mean creatinine was higher in the case group $(P=$ 0.027 ). Patients in the case group also required larger quantities of blood transfusion after the operation, when compared with the control group $(P=0.02)$. The hospital mortality did not differ between the analyzed groups. All these results can be seen in Table 1. (Table 1)

Logistic regression analysis revealed that independent risk factors for sternal wound infection were high BMI (odds ratio [OR] 1.15; CI 1.06-1.24), preoperative CRP concentration (OR 1.08; CI 1.01-1.16), long duration of cardiopulmonary bypass (OR 1.02; CI 1.01-1.03), intraoperative anemia (OR 0.97; CI 0.95-0.99) and postoperative CRP concentration (OR 1.10; CI 1.05-1.16). The probability of developing DSWI increases 1.15 times with every $1 \mathrm{~kg} / \mathrm{m} 2$ of BMI $(P=$ $0.001), 1.08$ times per $1 \mathrm{mg} / \mathrm{l}$ increase for preoperative CRP concentration $(P=0.036), 1.02$ times per 1 minute increase for duration of cardiopulmonary bypass $(P=0.001), 0.97$ times per $1 \mathrm{~g} / \mathrm{l}$ decrease for hemoglobin $(P=0.002)$, and 1.1 times per $10 \mathrm{mg} / \mathrm{l}$ increase for CRP concentration $(P<0.001)$. Results can be seen in Table 2. (Table 2)

\section{DISCUSSION}

Despite improvements over time with regard to morbidity, mortality and long-term survival, deep sternal wound infection (DSWI) continues to be a major complication after open-heart surgery [Lemaignen 2015; Sá 2017]. This is why it is important to identify possible risk factors for postoperative development of DSWI in patients undergoing coronary artery bypass grafting and valve replacement.

The incidence of deep sternal wound infection between 2017 January and 2018 December at the Department of Thoracic, Cardiac and Vascular Surgery of the Hospital of Lithuanian University of Health Sciences was 2.3\%. A total of 1936 patients underwent CABG and heart defect surgery, and 45 of them developed DSWI.

Both preoperative and postoperative CRP had a significant value on developing DSWI. This criterion is easily explained because a higher level of CRP means possible inflammation. 
Multivariate analysis identified three variables as highly significant independent predictors for the development of DSWI. We identified that higher BMI, anemia during surgery, and the duration of cardiopulmonary bypass are independent risk factors for the development of postoperative DSWI. Neither age nor sex or cardiac surgery in the patient's medical history was found to be significantly related to the prevalence of mediastinitis.

Higher BMI (>25 kg/m2) turned out to be one of the most important independent factors for the DSWI. Our data also show that the risk for mediastinitis increases by 1.15 times per additional kilogram body mass per square meter body surface. Numerous studies also have identified obesity as one of the strongest risk factors for postoperative DSWI [Ahmed 2011; Badawy 2014; Diez 2007, Gatti 2015; Lemaignen 2015; Meszaros 2016; Oliveira 2017; Risnes 2010; Sá 2017; Sá 2011; Schimmer 2016; Varma 2019; Vos 2018]. There are some factors that could explain why obesity is associated with the postoperative DSWI, and these factors have been discussed in previous studies. It is thought that the dose of prophylactic antibiotics is not corrected for the BMI of the patient, and the skin preparation could be more difficult [Sá

Table 1. Preoperative, operative, and postoperative data of the patients

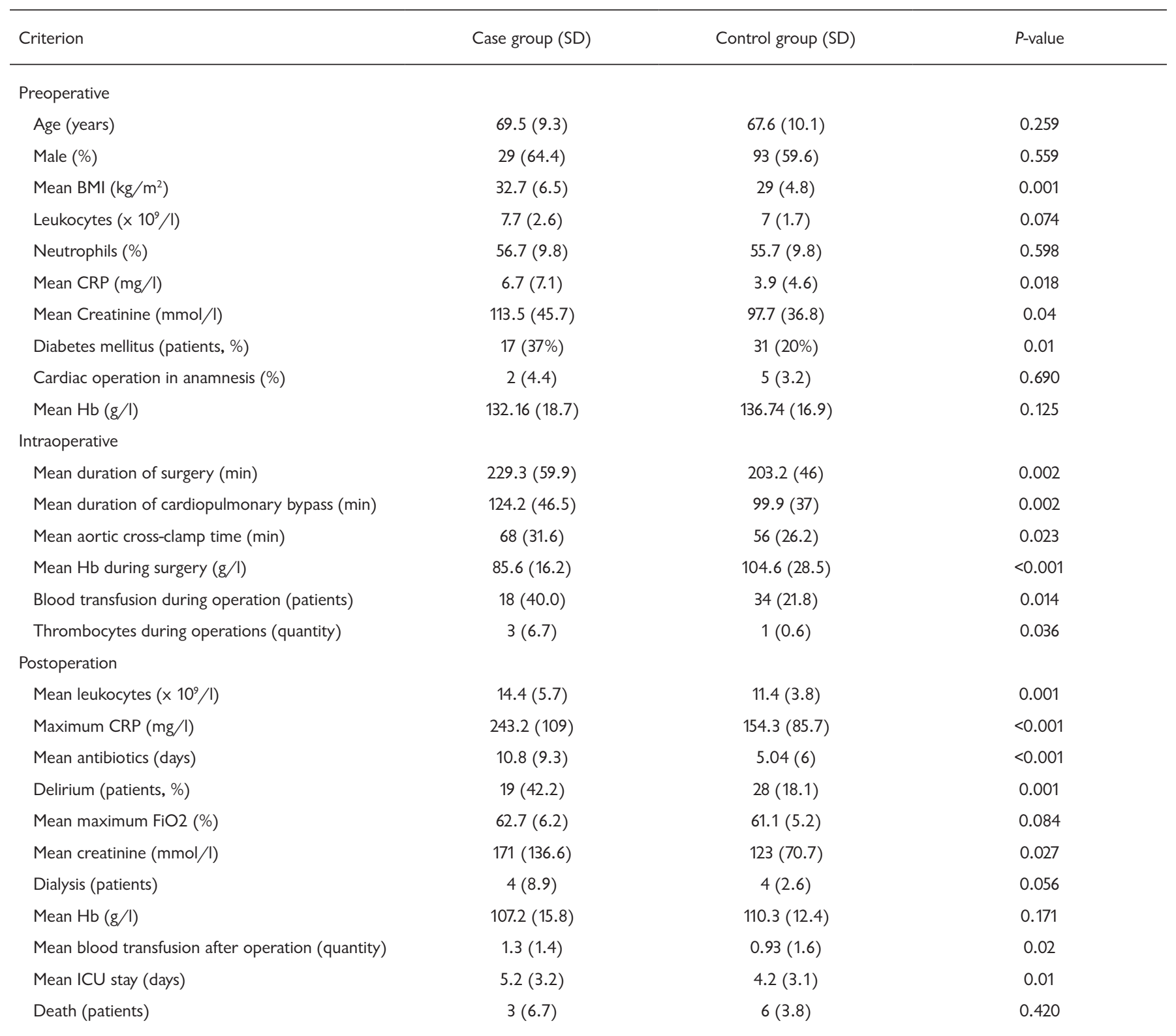

SD, standard deviation; BMI, body mass index; CRP, C reactive protein; FiO2, the fraction of inspired oxygen; Hb, hemoglobin; ICU, intensive care unit 
Table 2. Multivariate logistic model analysis results

\begin{tabular}{lccc}
\hline Variable & Odds ratio & Confidence interval & $P$-value \\
\hline $\mathrm{BMI}\left(\mathrm{kg} / \mathrm{m}^{2}\right)$ & 1.15 & $1.06-1.24$ & 0.001 \\
Preoperative CRP $(\mathrm{mg} / \mathrm{l})$ & 1.08 & $1.01-1.16$ & 0.036 \\
Duration of cardiopulmonary bypass $(\mathrm{min})$ & 1.02 & $1.01-1.03$ & 0.001 \\
Anemia during surgery (hemoglobin $(\mathrm{g} / \mathrm{l})$ & 0.97 & $0.95-0.99$ & 0.002 \\
Postoperative CRP $(\mathrm{mg} / \mathrm{l})$ & 1.10 & $1.05-1.16$ & $<0.001$ \\
\hline
\end{tabular}

BMI, body mass index; CRP, C-reactive protein

2017]. Additionally, fatty tissue does not contribute to wound healing [Diez 2007]. Weight reduction before surgery is recommended for all cases where BMI $>25 \mathrm{~kg} / \mathrm{m} 2$, but further research should be performed to discover what percentage of body mass a patient has to lose in order to minimize the incidence of infection following CABG [Ahmed 2011]. Minimizing weight cannot be achieved quickly before elective CABG. One study presumed that the independent association between obesity and SWI remains important after CABG but not after any valve surgery [Brunet 2020]. While obesity has been a risk factor for DSWI, some studies research that it is not associated with higher mortality after coronary artery bypass surgery, despite the ongoing infection [Crabtree 2004].

Intraoperative anemia was another important risk factor of DSWI in our study. We did not find an association between preoperative or postoperative anemia and DSWI. Some studies also stated that preoperative anemia was not associated with poor prognosis but there was a significant association between perioperative anemia and higher morbidity and mortality rate [Soh 2020; Vlot 2019]. Contrary to that, another study suggested that anemia assessed before CABG does independently increase the risk of postoperative morbidity and mortality [LaPar 2018]. One of the possible reasons for intraoperative anemia can be hemodilution because of the extracorporeal circuit. Intraoperative infusions for anesthesia management may be also caused by hemodilution. Optimizing pre-cardiopulmonary bypass volume could contribute to maintaining normal Hct and help avoid intraoperative hemodilution [Campbell 2008]. Since lower $\mathrm{Hb}$ is a modifiable risk factor that is linked to anemia during surgery, it is important to correct preoperative anemia whenever possible [Soh 2020].

Postoperative blood transfusion increases the risk to develop an infection after cardiac surgery as well as morbidity and mortality. This also was found in other research [Brunet 2020; Diez 2007; LaPar 2018; Lemaignen 2015; Oprea 2018; Risnes 2010; Schimmer 2016; Varma 2019].

The third independent predisposing factor in our study population was the duration of cardiopulmonary bypass (CPB). Prolonged CPB time also was identified as a possible risk factor for the DSWI in several studies [Kubota 2013; Schimmer 2016; Tewarie 2019]. Contrary to that, other authors did not observe a significant association between the duration of $\mathrm{CPB}$ and an increased risk of DSWI [Heilmann 2013; Oliveira 2017; Sá
2011]. Seese and colleagues observed that an increased number of complications after cardiac surgery occurs when $\mathrm{CPB}$ is applied longer than $100 \mathrm{~min}$ [Seese 2019]. One study found no difference in mediastinitis manifestation between operations whether CPB was used or not [Diez 2007]. A significant association between resternotomy and $\mathrm{CPB}$ was monitored after aortic valve replacement surgery [Chalmers 2014]. Based on our findings, the shortening of the duration of $\mathrm{CPB}$ could contribute to the prevention of postoperative DSWI.

Delirium represented $42 \%$ of people in the case group, where $\mathrm{CPB}$ time had a relevant association with infectious complications. Similarly, longer aortic cross-clamp time was found to be a risk factor for postoperative delirium in one study [Sugimura 2020], and a longer duration of extracorporeal circulation was associated with the presence of delirium in others [Jones 2019; Sanson 2018].

Diabetes mellitus, female gender, older age, and renal failure have been identified as risk factors for postoperative DSWI in previous studies [Ahmed 2011; Badawy 2014; Gatti 2015; Heilmann 2013; Kubota 2013; Lemaignen 2015; Ohira 2019; Risnes 2010; Sá 2011; Varma 2019]. Nevertheless, we have not observed any significant associations between diabetes mellitus, female gender, older age, renal failure, and a higher incidence of DSWI.

Poor glycemic control is a risk factor for developing DSWI [Gatti 2015]. Nonetheless, in our study, we did not include postoperative blood glycemic levels because according to our department protocol it is essential to maintain normoglycemia for all patients after CABG and heart defect surgery.

It is important to determine factors that are associated with DSWI in order to predict the patients that may have a higher risk of developing DSWI. The DSWI in the postoperative period of cardiovascular surgery is a serious complication and that is why several measures should be taken to avoid it [Diez 2007; Meszaros 2016].

\section{CONCLUSION}

This study demonstrates that higher BMI, preoperative and postoperative CRP concentration, anemia, and the duration of cardiopulmonary bypass are independent risk factors for the development of postoperative deep sternal wound infection. 


\section{REFERENCES}

Ahmed D, Cheema FH, Ahmed YI, Schaefle KJ, Azam SI, Sami SA, Sharif HM. 2011. Incidence and predictors of infection in patients undergoing primary isolated coronary artery bypass grafting: a report from a tertiary care hospital in a developing country. J Cardiovasc Surg (Torino) 52(1):99-104.

Badawy M, Shammari F, Aleinati T, Eldin M, Tarazi R, Alfadli J. 2014. Deep sternal wound infection after coronary artery bypass: How to manage? Asian Cardiovascular and Thoracic Annals 22(6):649-654.

Brunet A, et al. 2020. Obesity and Preoperative Anaemia as Independent Risk Factors for Sternal Wound Infection After Coronary Artery Bypass Graft Surgery with Pedicled (Non-Skeletonized) Internal Mammary Arteries: The Role of Thoracic Wall Ischemia? Vascular Health and Risk Management 16, 553-559.

Campbell JA, et al. 2008. Influence of Intraoperative Fluid Volume on Cardiopulmonary Bypass Hematocrit and Blood Transfusions in Coronary Artery Bypass Surgery. J Extra Corpor Technol 40:99-108.

Chalmers J, Pullan M, Mediratta N, Poullis M. 2014. A need for speed? Bypass time and outcomes after isolated aortic valve replacement surgery. Interactive CardioVascular and Thoracic Surgery 19:21-26.

Crabtree TD, et al. 2004. Multivariate Analysis of Risk Factors for Deep and Superficial Sternal Infection after Coronary Artery Bypass Grafting at a Tertiary Care Medical Center. Seminars in Thoracic and Cardiovascular Surgery 16: 53-61.

Diez C, Koch D, Kuss O, Silber RE, Friedrich I, Boergermann J. 2007. Risk factors for mediastinitis after cardiac surgery - A retrospective analysis of 1700 patients. Journal of Cardiothoracic Surgery, 2(1): 23.

Gatti G, Dell'Angela L, Barbati G, Benussi B, Forti G, Gabrielli M, Rauber E, Luzzati R, Sinagra G, Pappalardo A. 2015. A predictive scoring system for deep sternal wound infection after bilateral internal thoracic artery grafting. European Journal of Cardio-Thoracic Surgery 49(3):1-9.

Heilmann C, Stahl R, Schneider C, Sukhodolya T, Siepe M, Olschewski $\mathrm{M}$, et al. 2013. Wound complications after median sternotomy: a single-centre study. Interactive CardioVascular and Thoracic Surgery 16(5):643-648.

Jones D, et al. 2019. Predictors and Outcomes of Cardiac Surgery-Associated Delirium. A Single Centre Retrospective Cohort Study. Heart, Lung and Circulation 28:455-463.

Kubota H, Miyata H, Motomura N, Ono M, Takamoto S, Harii K, et al. 2013. Deep sternal wound infection after cardiac surgery. Journal of Cardiothoracic Surgery 8(1):32.

LaPar DJ, et al. 2018. Preoperative Anemia versus Blood Transfusion: Which is the Culprit for Worse Outcomes in Cardiac Surgery? Thorac Cardiovasc Surg 156:66-74.

Lemaignen A, Birgand G, Ghodhbane W, Alkhoder S, Lolom I, Belorgey S, et al. 2015. Sternal wound infection after cardiac surgery: incidence and risk factors according to clinical presentation. Clinical Microbiology and Infection 21(7):674,11-18.

Meszaros K, Fuehrer U, Grogg S, Sodeck G, Czerny M, Marschall J, Carrel T. 2016. Risk Factors for Sternal Wound Infection After Open Heart Operations Vary According to Type of Operation. Ann Thorac Surg 101(4):1418-25.

Ohira S, Miyata H, Yamazaki S, Numata S, Motomura N, Takamoto S, Yaku H. 2019. Deep sternal wound infection after bilateral internal thoracic artery grafting: Insights from a Japanese national database. The Journal of Thoracic and Cardiovascular Surgery: 157(1):166-173.

Oliveira F, Freitas L, Rabelo-Silva E, Costa L, Kalil R, Moraes M. 2017. Predictors of Mediastinitis Risk after Coronary Artery Bypass Surgery: Applicability of Score in 1.322 Cases. Arquivos Brasileiros de Cardiologia 9(3):207-212 .

Oprea AD, et al. 2018. Pre- and postoperative anemia, acute kidney injury, and mortality after coronary artery bypass grafting surgery: a retrospective observational study. Can J Anesth/J Can Anesth 65:46-59.

Risnes I, Abdelnoor M, Almdahl S, Svennevig J. 2010. Mediastinitis After Coronary Artery Bypass Grafting Risk Factors and Long-Term Survival. The Annals of Thoracic Surgery 89(5):1502-1509.

Sá M, Ferraz P, Soares A, Miranda R, Araújo M, Silva F, et al. 2017. Development and Validation of a Stratification Tool for Predicting Risk of Deep Sternal Wound Infection after Coronary Artery Bypass Grafting at a Brazilian Hospital. Brazilian Journal of Cardiovascular Surgery 32(1):1-17.

Sá M, Soares E, Santos C, Figueiredo O, Lima R, Escobar R, et al. 2011. Risk factors for mediastinitis after coronary artery bypass grafting surgery. Revista Brasileira de Cirurgia Cardiovascular 26(1):27-35.

Sanson G, et al. 2018. Delirium after cardiac surgery. Incidence, phenotypes, predisposing and precipitating risk factors, and effects. Heart Lung and Circulation 47:408-417.

Schimmer C, et al. 2016. Prevention of surgical site sternal infections in cardiac surgery: a two-centre prospective randomized controlled study. European Journal of Cardio-Thoracic Surgery 51:67-72.

Seese L, Sultan I, Gleason TG, Navid F, Wang Y, Thoma F, Kilic A. 2019. The Impact of Major Postoperative Complications on LongTerm Survival After Cardiac Surgery. The Annals of Thoracic Surgery 10(1):128-135.

Soh S, Shim J, Song J, Kang B, Kwak Y. 2020. Perioperative Nadir Hemoglobin Concentration and Outcome in Off-Pump Coronary Artery Bypass Surgery. A Retrospective Review. Circulation Journal 85(1):37-43.

Sugimura Y, et al. 2020. Risk and Consequences of Postoperative Delirium in Cardiac Surgery. Thorac Cardiovasc Surg 68:417-424.

Taylor A, Mitchell A, Mitchell I. 2012. A 15-year study of the changing demographics and infection risk in a new UK cardiac surgery unit. Interactive CardioVascular and Thoracic Surgery 15(3):390-394.

Tewarie L, Moza AK, Khattab MA, Autschbach R, Zayat R. 2019. Effective Combination of Different Surgical Strategies for Deep Sternal Wound Infection and Mediastinitis. Ann Thorac Cardiovasc Surg 25:102-110

Varma P, Gurram A, Krishna N, Vasudevan A, Baquero L, Jayant A. 2019. Female Gender is not a Risk Factor for Early Mortality after Coronary Artery Bypass Grafting. Ann Card Anaesth 22(2):187.

Vlot EA, et al. 2019. Intra-operative red blood cell transfusion and mortality after cardiac surgery. BMC Anesthesiol 19(1):65.

Vos R, Van Putte B, Kloppenburg G. 2018. Prevention of deep sternal wound infection in cardiac surgery: a literature review. Journal of Hospital Infection 100(4):411-420.

Yumun G, Erdolu B, Toktas F, Eris C, Ay D, Turk T, et al. 2014. Deep Sternal Wound Infection after Coronary Artery Bypass Surgery: Management and Risk Factor Analysis for Mortality. The Heart Surgery Forum 17(4):212. 